\title{
Kombinierte Wirkungen von Temperatur, Salzgehalt und Cadmium auf Entwicklung, Wachstum und Mortalität der Larven von Mytilus edulis aus der westlichen Ostsee
}

\author{
W. LeHNBerg \& H. Theede \\ Institut für Meereskunde an der Universität Kiel; \\ Disternbrooker Weg 20, D-2300 Kiel, Bundesrepublik Deutschland
}

\begin{abstract}
Combined effects of temperature, salinity and cadmium on development, growth and mortality of Mytilus edulis larvae from the western Baltic Sea. The response surface method was used to study the effects of temperature, salinity and $\mathrm{Cd}$ contamination on the development of fertilized eggs of Mytilus edulis from the western Baltic Sea to the veliger stage as well as on growth and cumulative mortality of a veliger population. The reactions observed differ considerably with reference to temperature and salinity. This is discussed in respect to the mode of life of the larvae and to the environmental conditions of the western Baltic Sea. $\mathrm{Cd}$ only slightly influences various temperature-dependent life functions, but strongly modifies those depending on salinity. The development optimum is shifted to higher salinities with increasing Cd concentrations of the medium, while that of survival and growth is shifted to lower salinities. These factor interactions modify the tolerance limits. In addition, the rates of factor interactions on the larval stages change with the degree of development. The trochophora stage proves to be most sensitive to the factors studied. A significant influence of cadmium on different life functions is found from concentrations of about $50 \mathrm{ppb}$ on.
\end{abstract}

\section{EINLEITUNG}

Nachdem die Itai-Itai-Krankheit mit ihren fatalen Auswirkungen auf den Menschen als Cadmiumvergiftung diagnostiziert worden ist (Douglas-Wilson, 1972), gilt Cadmium als eines der stärksten vom Menschen in die Umwelt gebrachten Gifte. Da es bereits in geringen Konzentrationen (ppb-Bereich) schädigend auf Organismen wirken kann, spielt es eine wichtige Rolle in der ökologisch-toxikologischen Forschung.

Der Cadmiumgehalt der Ostsee wird von Kremling (1973) mit 0,1-0,2 ppb angegeben. In den trockenen Tonanteilen von Sedimenten der Deutschen Bucht wurden Konzentrationen von 2 ppm gemessen (Förstner \& Reinbeck, 1974), was dafür spricht, daß sich das Cadmium an partikuläre Substanzen anlagert und mit diesen sedimentiert (Friberg et al., 1976). Solche Stoffe können jedoch von Filtrierern als potentielle Nahrung zurückgehalten werden und zu den stellenweise sehr hohen Cd-Gehalten in Miesmuscheln (Mytilus edulis) beitragen (Theede et al., 1979a). 
Die letalen Cadmiumkonzentrationen unterscheiden sich in starkem Maße bei verschiedenen Tiergruppen (Theede, 1979). Hinzu kommt, daß die akute Toxizität von Cadmium auch von anderen gleichzeitig wirkenden abiotischen Faktoren stark modifiziert werden kann (Rosenberg \& Costlow, 1976; Theede et al., 1979b; Westernhagen et al., 1975). Um Anhaltspunkte über mögliche ökologische Auswirkungen erhöhter Cadmiumkonzentrationen in Lebensräumen mit starken Schwankungen der abiotischen Umweltbedingungen (Gezeitenregion, Åstuare, Ostsee) zu erhalten, erscheint es deshalb unumgänglich, diese Wechselwirkungen zu berücksichtigen. Das ist das Ziel der vorliegenden Arbeit.

Adulte Individuen von Mytilus edulis erweisen sich gegenüber zahlreichen natürlichen Umweltfaktoren als euryök (Schlieper \& Theede, 1974; Theede, 1965, 1975). Sie sind auch gegenüber den toxischen Einflüssen von Schwermetallen verhältnismäßig tolerant (Theede, 1979). Die Larven sind jedoch wesentlich empfindlicher gegenüber verschiedenen Außenfaktoren (Bayne, 1965; Brenko \& Calabrese, 1969). Da die Resistenzgrenzen der empfindlicheren Fortpflanzungs- und Entwicklungsstadien wesentlich wichtiger für die Existenz der Art in ihrem Lebensraum sind als die der resistenten Adulten, wurden die vorliegenden Untersuchungen über die Toxizität von Cadmium an den Larven von $M$. edulis durchgeführt.

Mit der "response surface"-Methodik (Box \& Wilson, 1951) liegt ein Verfahren vor, komplexe Zusammenhänge zwischen den Reaktionen eines Organismus und mehreren gleichzeitig einwirkenden Faktoren mit einem vertretbaren Aufwand experimentell zu analysieren. Mit Hilfe dieser Methodik werden in der vorliegenden Arbeit Entwicklung, Wachstum und Mortalităt der Larven von Mytilus edult's aus der westlichen Ostsee in Abhängigkeit von den beiden dominierenden Faktoren Temperatur und Salzgehalt sowie der Belastung des Meerwassers mit Cadmium untersucht.

\section{MATERIAL UND METHODE}

Etwa 6-10 cm große Exemplare der Miesmuschel Mytilus edulis wurden im Spätwinter und Frühjahr in der Eckernförder Bucht (westliche Ostsee) gedretscht und in Aquarien bei $5{ }^{\circ} \mathrm{C}$ und $18-20 \% \mathrm{~S}$ gehältert. Nach der Methode von Loosanoff \& Davis (1963) wurden sie zur Abgabe von Eiern und Spermien gebracht. Die vorliegenden Versuche wurden mit den Nachkommen eines Elternpaares durchgeführt, mit Ausnahme der bifaktoriellen Vorversuche.

Jeweils dreitausend bis füntausend Tiere wurden in Kristallisierschalen in $100 \mathrm{ml}$ Versuchswasser aufgezogen. Als Kulturmedium diente Ostseewasser, das mit Meersalz (tropic marine neu) auf etwa $40 \% \mathrm{~S}$ aufgesalzen und mit demineralisiertem Wasser auf die erforderlichen Salzgehalte verdünnt wurde. Das Wasser wurde lediglich papierfiltriert, da eine völlig bakterienfreie Filtration nicht nötig war. Der Cadmiumblindwert lag bei 0 bis $3 \mathrm{ppb}$. Die Kulturschalen befanden sich in temperierten Bädern.

Eine angesäuerte $1-\%, \mathrm{Cd}^{2+}$-Stammlösung wurde aus $\mathrm{CdCl}_{2}$ (Merck, Titrisol) hergestellt, vor Anwendung auf 100 ppm verdünnt und mit einer Mikropipette in entsprechenden Mengen dem Versuchswasser zugefügt. Die Konzentrationen schwanken um maximal $10 \%$ vom Mittelwert. Sie wurden mittels AAS (Beckmann) bestimmt.

Als Nahrung diente Dunaliella marina. Nach jedem Wasserwechsel wurden etwa 
$1,5 \times 10^{5}$ Zellen zugegeben. Die mit diesem Futter erzielten Wachstumsraten liegen im unteren Bereich der von Bayne (1965) für verschiedene Futteralgen erzielten optimalen Wachstumsraten.

Für die Versuche mit Trochophora-Larven wurden die sich schnell entwickelnden, befruchteten Eier innerhalb von 30 bis $60 \mathrm{~min}$ an die Versuchsbedingungen angepaßt. Dann wurde Cadmium zugesetzt (Zeitpunkt 0 ). Für Versuche, die mit späteren Stadien begannen, wurden die Tiere zunächst über etwa $80 \mathrm{~h}$ bei $15^{\circ} \mathrm{C} / 21 \% \mathrm{~S}$ aufgezogen und dann zunächst $6 \mathrm{~h}$ schrittweise an die Versuchsbedingungen angepaßt. $24 \mathrm{~h}$ später wurden sie nach einer Kontrollzählung mit Cadmium versetzt (Zeitpunkt 0 ).

Wasserwechsel erfolgte montags, mittwochs und freitags. Vor jedem Wasserwechsel wurde das Verhältnis toter : lebender Tiere bestimmt und die Länge der mit einem $50 \mu$ Netz abfiltrierten, lebenden Tiere gemessen.

Die Versuche wurden nach der "response surface"-Methode geplant und ausgewertet. Als experimentelles Design diente ein modifiziertes, orthogonales, zusammengesetztes Design, $2\left(2^{\mathrm{k}}\right)+2(\mathrm{k})+2$ nach Alderdice $\&$ Thomson (1974). Sieben verschiedene Werte der Faktoren wurden entsprechend der Tabelle 1 kombiniert. Als nichtlineare Approximationsfunktion diente die Gleichung

$$
\begin{aligned}
& y^{c}=b_{o}+b_{1} x_{1}{ }^{a_{1}}+b_{2} x_{2}{ }^{a_{2}}\left(+b_{3} x_{3}{ }^{a_{3}}\right)+b_{11} x_{1}{ }^{2 a_{1}}+b_{22} x_{2}{ }^{2 a_{2}}\left(+b_{33} x_{3}{ }^{2 a 3}\right)+b_{12} x_{1}{ }^{a_{1}} x_{2}{ }^{a_{2}} \\
& \left(+b_{13} x_{1}{ }^{a_{1}} x_{3}{ }^{a_{3}}+b_{23} x_{2}{ }^{a_{2}} x_{3}{ }^{a_{3}}\right)
\end{aligned}
$$

für zwei und drei () Faktoren, sowie die entsprechenden linearen Regressionsgleichungen mit $c, a_{1}, a_{2}, a_{3}=1$. Die Berechnung der Parameter $(a, b$ und $c$ ) erfolgte mit der maximum likelihood-Methode (Lindsey et al., 1970) und wurde an der Rechenanlage der Universität Kiel nach einem Fortran Programm von Lindsey \& Sandnes (1970) durchgeführt. Das Rechenprogramm liefert u. a. zu den Parameterwerten und Zentrumskoordinaten der Reaktionsfläche deren relative maximum likelihood-Verteilungen, Varianzen, Kovarianzen, Varianzanalysen der linearen und nichtlinearen Regression, eine kanonische Analyse mit einem Vergleich der gemessenen und den nach der Regression berechneten Daten

Tabelle 1

Das „experimental design“ für drei Faktoren ( $\mathrm{T}=$ Temperatur in ${ }^{\circ} \mathrm{C}, \mathrm{S}=$ Salzgehalt in $\%$ und $\mathrm{Cd}=$

\begin{tabular}{|c|c|c|c|c|c|c|c|}
\hline Nr. & $\mathrm{T}$ & $S$ & $\mathrm{Cd}$ & Nr. & $\mathrm{T}$ & $\mathrm{S}$ & $\mathrm{Cd}$ \\
\hline 1 & 10 & 32 & 195 & 15 & 17,5 & 26,5 & 156,8 \\
\hline 2 & 10 & 32 & 42 & 16 & 17,5 & 26,5 & 80,3 \\
\hline 3 & 10 & 10 & 195 & 17 & 17,5 & 15,5 & 156,8 \\
\hline 4 & 10 & 10 & 42 & 18 & 17,5 & 15,5 & 80,3 \\
\hline 5 & 12,5 & 26,5 & 156,8 & 19 & 20 & 32 & 195 \\
\hline 6 & 12,5 & 26,5 & 80,3 & 20 & 20 & 32 & 42 \\
\hline 7 & 12,5 & 15,5 & 156,8 & 21 & 20 & 10 & 195 \\
\hline 8 & 12,5 & 15,5 & 80,3 & 22 & 20 & 10 & 42 \\
\hline 9 & 15 & 21 & 118,5 & 23 & 22,4 & 21 & 118,5 \\
\hline 10 & 15 & 21 & 118,5 & 24 & 7,7 & 21 & 118,5 \\
\hline 11 & 15 . & 4,8 & 118,5 & & & & \\
\hline 12 & 15 & 37,3 & 118,5 & & & & \\
\hline 13 & 15 & 21 & 3,8 & & & & \\
\hline 14 & 15 & 21 & 233,3 & & & & \\
\hline
\end{tabular}
$\mathrm{Cd}^{2+}$-Konzentration in $\mathrm{ppb}$ ) 
sowie Isoliniendiagramme für beliebige Schnitte durch den Faktorenraum.

Zur Interpretation von Reaktionsflächen sei auf Alderdice (1973) und Lehnberg (1974) verwiesen. Grundsätzlich bedeutet ein zu einer Faktorenachse paralleler Verlauf von Isolinien: kein Einfluß dieses Faktors, senkrechter Verlauf: maximaler Einfluß, und eine mehr oder weniger diagonal verlaufende Isolinie oder Achse einer Reaktionsfläche drückt eine Wechselwirkung von zwei Faktoren aus.

$\mathrm{Da}$ die Größen der Tiere innerhalb der Kulturen nicht normal verteilt waren, wurden Mediane (neben Momenten, Kurtosis etc.) berechnet. Um die zufälligen Schwankungen zeitlich aufeinanderfolgender Werte von kumulierender Mortalität und mittlerer Größe der Veligerlarven einer Kultur auszugleichen, wurden gleitende Durchschnitte von drei zeitlich aufeinanderfolgenden Werten berechnet, wodurch sich gebrochene Zeitwerte ergeben können. Aufgeführt wird im folgenden die Regression, die die bessere Approximation liefert.

\section{ERGEBNISSE}

\section{Entwicklung der Trochophora}

In Vorversuchen wurde zunächst geprüft, ob die gewählten Grenzen und Faktorenkombinationen von Temperatur und Salzgehalt für die Analyse verwertbare Ergebnisse erwarten lassen würden. $24 \mathrm{~h}$ nach der Befruchtung (=Versuchsbeginn) hatte bei den Kombinationen $15{ }^{\circ} \mathrm{C} / 4,75 \% \mathrm{~S}$ und $15{ }^{\circ} \mathrm{C} / 37,25 \% \mathrm{~S}$ keine Entwicklung stattgefunden. Unter allen anderen Bedingungen wuchsen mit unterschiedlicher Entwicklungsgeschwindigkeit mehr oder weniger lebhafte Tiere heran. Jedoch wurden Mißbildungen in Form ungeordneter Furchungsteilungen bei $10 \% \mathrm{~S}$ in Kombination mit $10^{\circ} \mathrm{C}$ und $20^{\circ} \mathrm{C}$ beobachtet. Die Abhängigkeit ließ sich zu diesem Zeitpunkt noch nicht mathematisch beschreiben.

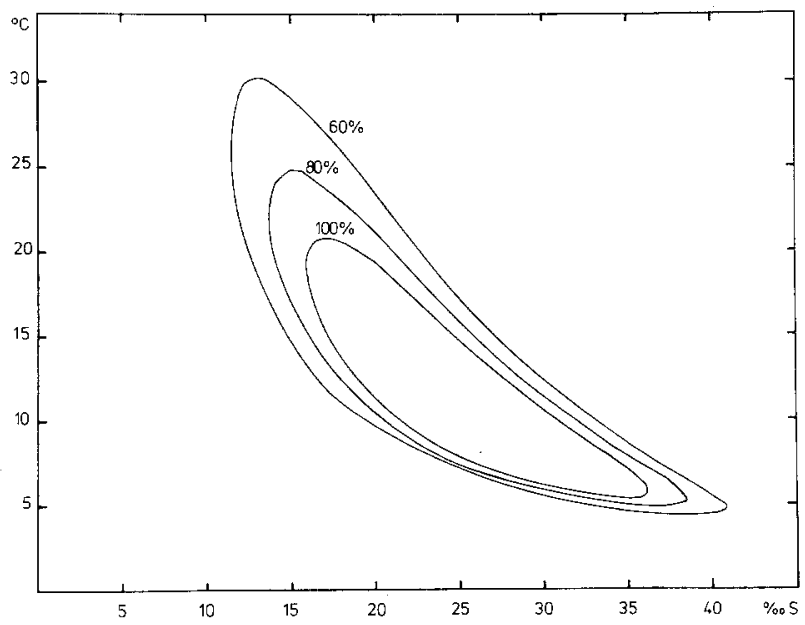

Abb. 1: Anteil der Trochophora-Larven an der Gesamtpopulation $48 \mathrm{~h}$ nach der Befruchtung unter dem Einfluß von Temperatur und Salzgehalt 
Nach $48 \mathrm{~h}$ hatte sich der Einfluß von Temperatur und Salzgehalt auf die Entwicklungsrate soweit manifestiert, daß er über die mathematische Analyse in der Form

$$
\begin{aligned}
& \mathrm{y}^{0,165}=-6,631+50,950 \mathrm{~T}^{-0,276}-0,350 \mathrm{~S}^{1,0}-82,559 \mathrm{~T}^{-0,552}-0,0075 \mathrm{~S}^{2,0} \\
&+1,395 \mathrm{~T}^{-0,276} \mathrm{~S}^{1,0}
\end{aligned}
$$

zum Ausdruck kommt ( $\mathrm{y}=\%$ entwickelte Tiere, $\mathrm{T}=$ Temperatur in ${ }^{\circ} \mathrm{C}, \mathrm{S}=$ Salzgehalt in \%o). Da die linearen Glieder nach der Varianzanalyse eine geringe Signifikanz aufweisen, könnten sie entfallen, ohne das Modell ernstlich zu stören. Die Reaktionsfläche (Abb. 1) zeigt ein Zentrum bei etwa $25 \% \mathrm{~S}$ und $10^{\circ} \mathrm{C}$, um das sich ein schmaler sichelförmiger Bereich legt, in dem eine optimale Entwicklung stattfindet. Geht man vom Einfluß der einzelnen Faktoren aus, so ist die tolerierte Temperatur- und Salzgehaltsspanne verhältnismäßig eng. Zieht man jedoch die Wechselwirkung der Faktoren in Betracht, so läßt sich bei entsprechender Änderung beider Faktoren eine breite Zone überstreichen, innerhalb derer sich die Population mit einem hohen Prozentsatz entwickelt.

$72 \mathrm{~h}$ nach Versuchsbeginn hat sich die Struktur der Reaktionsfläche verändert (Abb. 2). Die Regressionsgleichung lautet

$$
\begin{gathered}
\mathrm{y}^{0,21}=-2,809+117,0 \mathrm{~T}^{-1,422}+0,230 \mathrm{~S}^{1,119}-2258,42 \mathrm{~T}^{-2,844}-0,004 \mathrm{~S}^{2,238} \\
+1,408 \mathrm{~T}^{-1,422} \mathrm{~S}^{1,119}
\end{gathered}
$$

Die Gesamtpopulation besteht jetzt bereits zu einem großen Teil aus Veligerlarven. Das Optimum der Entwicklung liegt bei $23,5 \% \mathrm{~S}$ und $10^{\circ} \mathrm{C}$ und damit nahezu unverändert im Faktorenfeld.

Die innerhalb von $24 \mathrm{~h}$ erfolgten Veränderungen kommen in den mathematischen Darstellungen über die Zunahme der Absolutbeträge der Parameter zum Ausdruck und entsprechen einem Anwachsen der Einflüsse der Faktoren auf die Entwicklung (Die Variation eines Faktors führt jetzt zu stärkeren Veränderungen in der Entwicklungsrate als zum 48-h-Zeitpunkt). Ferner erfolgt eine Umkehrung des Vorzeichens des linearen $\mathrm{S} \%$ Gliedes, was zu Veränderungen in der Form der Reaktionsfläche führt. In Abb. 2 ist der

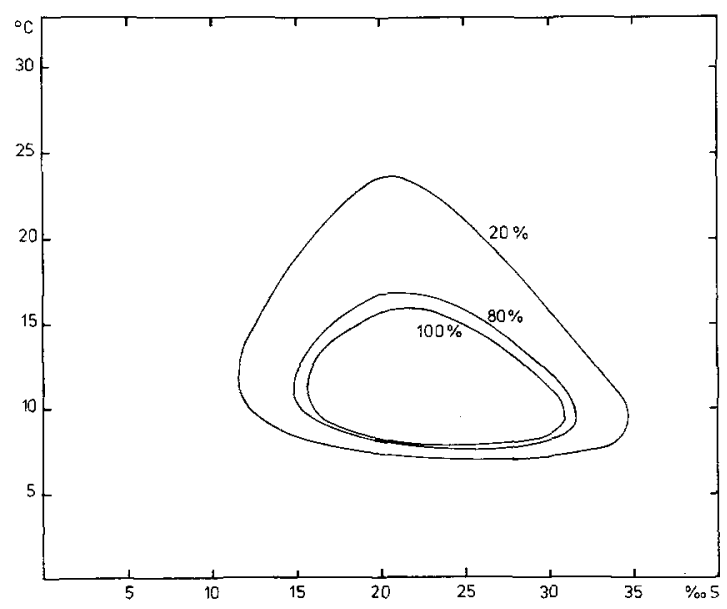

Abb. 2: Anteil der Trochophora- und Veliger-Larven an der Gesamtpopulation $72 \mathrm{~h}$ nach der Befruchtung unter dem Einfluß von Temperatur und Salzgehalt 
oberhalb von $15{ }^{\circ} \mathrm{C}$ befindliche Teil der "Sichel" nahezu verschwunden. Hier endet die rasche Entwicklung in einer hohen Mortalitätsrate. Unterhalb von $20 \% \mathrm{~S}$ zwischen etwa $8{ }^{\circ} \mathrm{C}$ und $15^{\circ} \mathrm{C}$ hat sich die Reaktionsfläche ausgedehnt; dies besagt, daß hier die Entwicklung langsam abläuft.

\section{Entwicklung zur Veliger}

Die folgenden Versuche schließen das Cadmium als dritten Faktor in die Versuche ein. Durch hohe Sterblichkeitsraten für verschiedene Faktorenkombinationen bestätigten sie den schmalen Entwicklungsspielraum, der der Trochophora zur Verfügung steht. Eine Datenanalyse der Entwicklungsgeschwindigkeit in den ersten Tagen lieferte keine sinnvollen Ergebnisse. Die Versuche wurden deshalb mit etwa $80 \mathrm{~h}$ alten (bei $15^{\circ} \mathrm{C}$ und $21 \% \mathrm{~S}$ aufgezogenen) Larven begonnen. $\mathrm{Zu}$ diesem Zeitpunkt setzt sich die Ausgangspopulation aus Larvenstadien zusammen, die eine beginnende bis volle Beschalung und Velum zeigen (vgl. Field, 1922; Abb. 183 f-h). Das Ausgangsverhältnis Veliger : Trochophora liegt bei
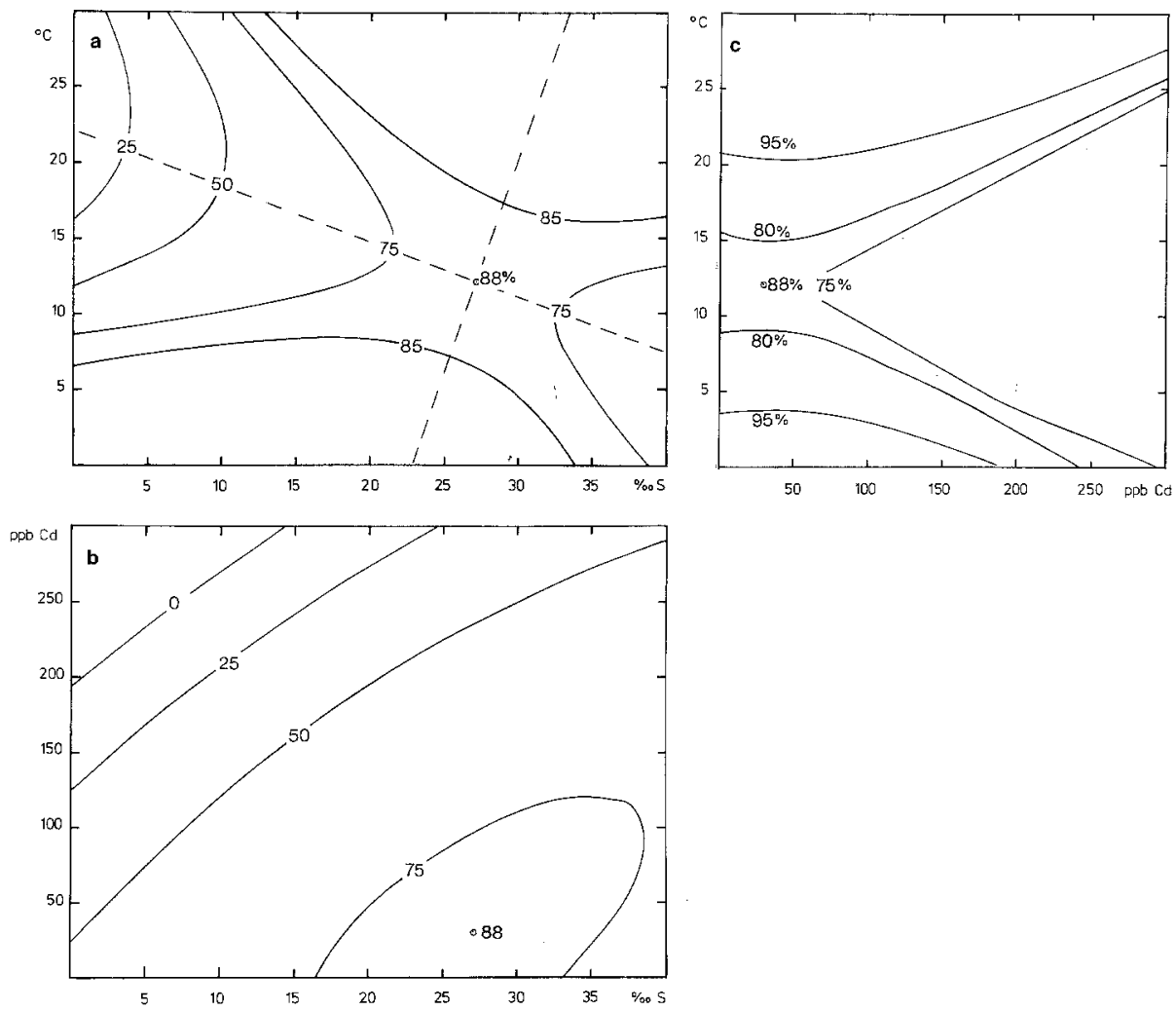

Abb. 3: Anteil der Veliger-Larven an der Gesamtpopulation nach 6 Tagen unter dem Einfluß von Temperatur, Salzgehalt und Cadmium (Ausgangsverhältnis Trochophora : Veliger $=3: 2$ ), (a) T-SAbhängigkeit bei $30 \mathrm{ppb} \mathrm{Cd}$ im Medium, (b) Cd-S-Abhängigkeit bei $12{ }^{\circ} \mathrm{C}$, (c) T-Cd-Abhängigkeit bei $27 \%$ S. (Die Schnitte gehen durch das Zentrum des Reaktionskörpers) 
etwa $40: 60$ (2:3). Etwa sechs Tage nach Versuchsbeginn wird der Stand der Entwicklung der Trochophora- zur Veligerlarve unter Berücksichtigung des Anteils der Veliger an der Gesamtpopulation (Trochophora und Veliger) erfaßt (Abb. 3). Die Abhängigkeit läßt sich mit der Regressionsgleichung

$$
\begin{gathered}
y=170,04-12,67 \mathrm{~T}+0,974 \mathrm{~S}-0,159 \mathrm{Cd}+0,25 \mathrm{~T}^{2}-0,04 \mathrm{~S}^{2}-0,00065 \mathrm{Cd}^{2}+0,243 \mathrm{TS} \\
+0,0009 \mathrm{TCd}+0,0069 \mathrm{SCd}
\end{gathered}
$$

( $y=\%$ Veliger) beschreiben. Abb. 3 zeigt die zweifaktoriellen Schnitte durch das Zentrum des Reaktionskörpers im Punkte $12^{\circ} \mathrm{C} / 27 \% \mathrm{~S} / 30 \mathrm{ppb}$ Cd. Der geringe Cadmiumwert erlaubt es, die temperatur-salzgehaltsabhängige Weiterentwicklung der Trochophora zur Veliger (Abb. 3a) mit der Entwicklung zur Trochophora (Abb. 1, 2) zu vergleichen. Trotz der unterschiedlichen geometrischen Interpretation der neuen Versuchsdaten im Vergleich zu den bisher dargestellten, lassen sich doch Gemeinsamkeiten herausstellen. Das Optimum liegt weiterhin bei etwa $25 \% \mathrm{~S}$ und geringfügig höherer Temperatur $\left(12^{\circ} \mathrm{C}\right)$. Die hyperbelförmigen Isolinien schließen etwa das in Abb. 2 dargestellte Dreieck ein. Die Situation läßt sich nur in Verbindung mit der bisher beschriebenen Trochophoraentwicklung und den noch zu erörternden Mortalitätsraten der Veliger erklären. So sind die hohen Prozentsätze entwickelter Veliger bei niedrigen Temperaturen nicht auf eine beschleunigte Entwicklung zurückzuführen, sondern darauf, daß die Mortalitätsraten der Trochophora mit sinkenden Temperaturen schneller zunehmen als die der Veliger. Der hohe Veligeranteil bei hohen Temperaturen und Salzgehalten erklärt sich jedoch ausschließlich durch hohe Sterblichkeitsraten der Trochophora. Für weiterentwickelte Tiere erweist sich dieser Bereich des Faktorenfeldes als günstig (siehe Abb. 10a).

In dieser Ubergangsphase von der Trochophora zur Veliger verändert sich die Wechselwirkung von Temperatur und Salzgehalt, was zu einer Verlagerung der tolerierten Temperatur-Salzgehaltsbereiche führt. Ein Maß für die Wechselwirkung ist die Größe des Winkels, den die Achse der Reaktionsfläche mit der entsprechenden Koordinatenachse bildet. In Abb. 1 verläuft die Achse der Reaktionsfläche etwa durch das Zentrum und durch die Spitzen der "Sichel". Mit der ihr zugehörigen Salzgehaltsachse bildet sie einen großen, positiven (im Uhrzeigersinn gedrehten) Winkel, was einer starken Wechselwirkung entspricht und zu schmalen Toleranzbereichen führt (siehe "Entwicklung der Trochophora"). Mit der Entwicklung der Veliger nimmt diese Wechselwirkung stark ab (Verkleinerung des Winkels), was zu größeren Toleranzbereichen führt, insbesondere für niedrige Salzgehalte oder hohe Temperaturen, jeweils vom Zentrum ausgehend.

Die Wirkung des Cadmiums auf die temperatur- und salzgehaltsabhängige Entwicklung geht aus Abb. 3 (b und c) hervor. Zwischen dem Cadmium und dem Salzgehalt besteht eine starke Wechselwirkung, die ein negatives Vorzeichen trägt, da die Reaktionsfläche entgegen dem Uhrzeigersinn gedreht ist. Mit zunehmender Cadmiumkonzentration wird die optimale Entwicklung auf höhere Salzgehalte hin verlagert, während sich bei niedrigen Salzgehalten der entwicklungshemmende Einfluß des Schwermetalls verstärkt. Mit der Temperatur besteht keine Wechselwirkung. Ihr Einfluß überwiegt deutlich den des Cadmiums. Auf die Dimension der Isolinien wurde bereits oben hingewiesen. 


\section{Kumulierende Mortalität von Veligerlarven}

Etwa sechs Tage nach Versuchsbeginn liefert die Datenanalyse eine maximale Úberlebensrate $(>90 \%)$ bei $16^{\circ} \mathrm{C}$ und $33 \%$ S ohne Cd-Zusatz im Medium. Beide Werte liegen höher als die Koordinaten für optimale Entwicklung bis zu diesem Zeitpunkt. Für $150 \mathrm{ppb}$ $\mathrm{Cd}$ im Medium befindet sich das Zentrum der Reaktionsfläche bei etwa $10^{\circ} \mathrm{C}$ und $25 \% \mathrm{~S}$ (Abb. 4a). Die Verlagerung des Mortalitätsminimums mit ansteigender Cadmiumkonzentration deutet auf eine Wechselwirkung des Schwermetalls sowohl mit der Temperatur als auch dem Salzgehalt hin. Die Reaktionsfläche läßt eine ausgeprägte Toleranz in einem weiten Salzgehaltsbereich (von 5 bis über $30 \% \mathrm{~S}$ ) im Bereich des Temperaturoptimums (um $10^{\circ} \mathrm{C}$ ) erkennen. Bei diesen Bedingungen ist jedoch die Weiterentwicklung der Trochophora deutlich gehemmt. Die Sterblichkeitsrate nimmt erst bei supraoptimalen Salzgehalten oder suboptimalen Temperaturen zu. Eine leichte, zu einem späteren Zeitpunkt (Abb. 5a) deutlicher hervortretende Ausbuchtung der Isolinien zwischen 20 und $35 \% \mathrm{~S}$ oberhalb des Temperaturoptimums, $d$. h. die leicht günstigeren Überlebenschan-
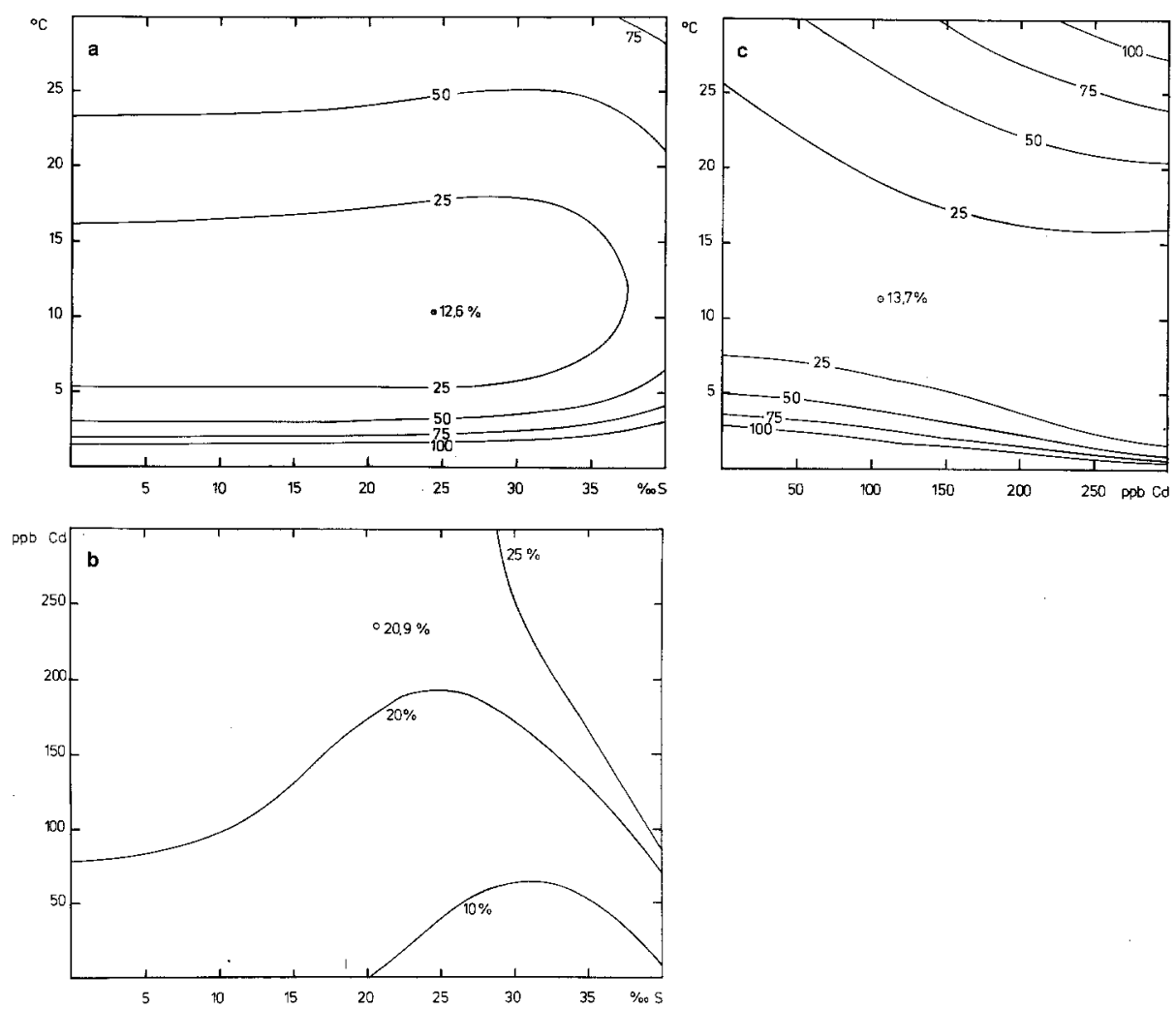

Abb. 4: Kumulierende Mortalität von Veliger-Larven 5,7 Tage nach Versuchsbeginn unter dem Einfluß von Temperatur, Salzgehalt und Cadmium, (a) T-S-Abhängigkeit bei $150 \mathrm{ppb} C d$ im Medium, (b) Cd-S-Abhängigkeit bei $15^{\circ} \mathrm{C}$, (c) T-Cd-Abhängigkeit bei $21 \% \mathrm{~S}$ 
cen in diesem Teil des Faktorenfeldes, sind teilweise für den hohen Veligeranteil bei der Trochophora-Veliger-Entwicklung verantwortlich.

Die erwähnte gegenseitige Beeinflussung von Salzgehalt und Cd-Konzentration verschiebt bei zunehmendem Cadmiumgehalt das Mortalitätsminimum in den Bereich niedrigerer Salzgehalte. Diese Art der Wechselwirkung zeigt einen völlig andersartigen Effekt als bei der Trochophora-Veliger-Entwicklung. Bemerkenswert ist, daß hier oberhalb des Salzgehaltsoptimums der Cadmiumeinfluß stärker als unterhalb desselben ist (Abb. $4 \mathrm{~b}$ ). Die Wechselbeziehungen zwischen den Wirkungen der Schwermetallkonzentration und der Temperatur führen bei zunehmender Cd-Konzentration zu einer Verlagerung des Temperaturoptimums zu niedrigeren Temperaturen hin. Die Wirkung der Temperatur überwiegt deutlich die des Schwermetalls, das auf Grund der Art der Wechselwirkung oberhalb des Temperaturoptimums die Mortalitätsraten beeinflußt.

Nach sechstägiger Einwirkung von $150 \mathrm{ppb} C d$ steigt die Mortalitätsrate für $15^{\circ} \mathrm{C}$ innerhalb einer weiten Salzgehaltsspanne ( 5 bis über $30 \% \mathrm{~S}$ ) auf das mehr als Doppelte der Rate ohne Cadmiumbelastung (von etwa $10 \%$ auf $25 \%$; Abb. 4a, b, c). Eine vergleichbare Wirkung ruft eine Temperatur von $25^{\circ} \mathrm{C}$ (ohne Cadmiumbelastung) hervor.
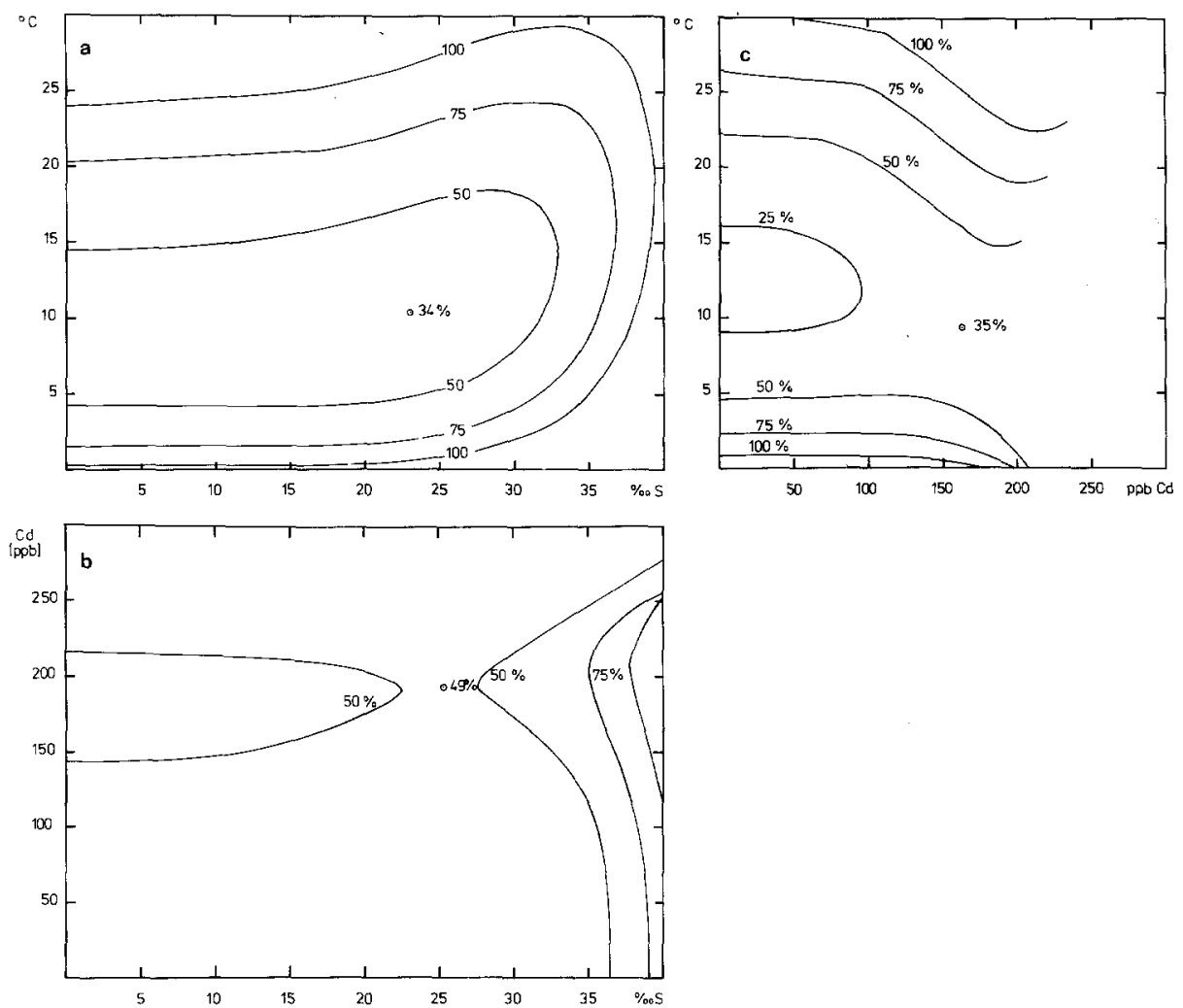

Abb. 5: Kumulierende Mortalität von Veliger-Larven 10,3 Tage nach Versuchsbeginn unter dem Einfluß von Temperatur, Salzgehalt und Cadmium, (a) T-S-Abhängigkeit bei $150 \mathrm{ppb}$ Cd im Medium, (b) Cd-S-Abhängigkeit bei $15^{\circ} \mathrm{C}$, (c) T-Cd-Abhängigkeit bei $21 \%$ 
Mit zusätzlich 150 ppb Cd erreicht die Mortalitätsrate bereits die 50\%-Marke (Abb. 4c).

Bis zum 10. Tag nach Versuchsbeginn bleibt die Art der Wirkungen der drei Faktoren auf die kumulierende Mortalität weitgehend unverändert. Jedoch wächst die Kapazität des Raktionskörpers auf das Zwei- bis Dreifache an, was insbesondere einer zunehmenden letalen Wirkung des Cadmiums oberhalb von etwa 100 bis $150 \mathrm{ppb}$ zuzuschreiben ist (Abb. 5). Innerhalb der erwähnten Salzgehaltsspanne und $150 \mathrm{ppb} \mathrm{Cd}$ im Medium liegt jetzt die 50- \%-Marke im günstigen Temperaturbereich von $15{ }^{\circ} \mathrm{C}$.

Das Auseinanderfließen der Isolinien in Abb. 5 (b und c) bei höheren Cadmiumkonzentrationen als $200 \mathrm{ppb}$ darf nicht als zunehmende Toleranz gegenüber diesen hohen Konzentrationswerten interpretiert werden. Der höchste verwendete Cadmiumgehalt im Medium betrug $233 \mathrm{ppb}$, so daß die Reaktionsflächen nur bis zu dieser Konzentration Gültigkeit haben, und die über diese Grenze hinausreichende Darstellung lediglich mögliche Tendenzen vermitteln kann. Etwa an Hand der $50 \%$ Isolinie in der Abb. 5b ist leicht einzusehen, daß die unteren Abschnitte über das 49-\%-Zentrum zusammenlaufen und unter Berücksichtigung der Verhältnisse im gültigen Teil der Reaktionsfläche ein entsprechender Verlauf höher dimensionierter Isolinien oberhalb von $200 \mathrm{ppb}$ in dieser und in Abb. $5 c$ erwartet werden darf.
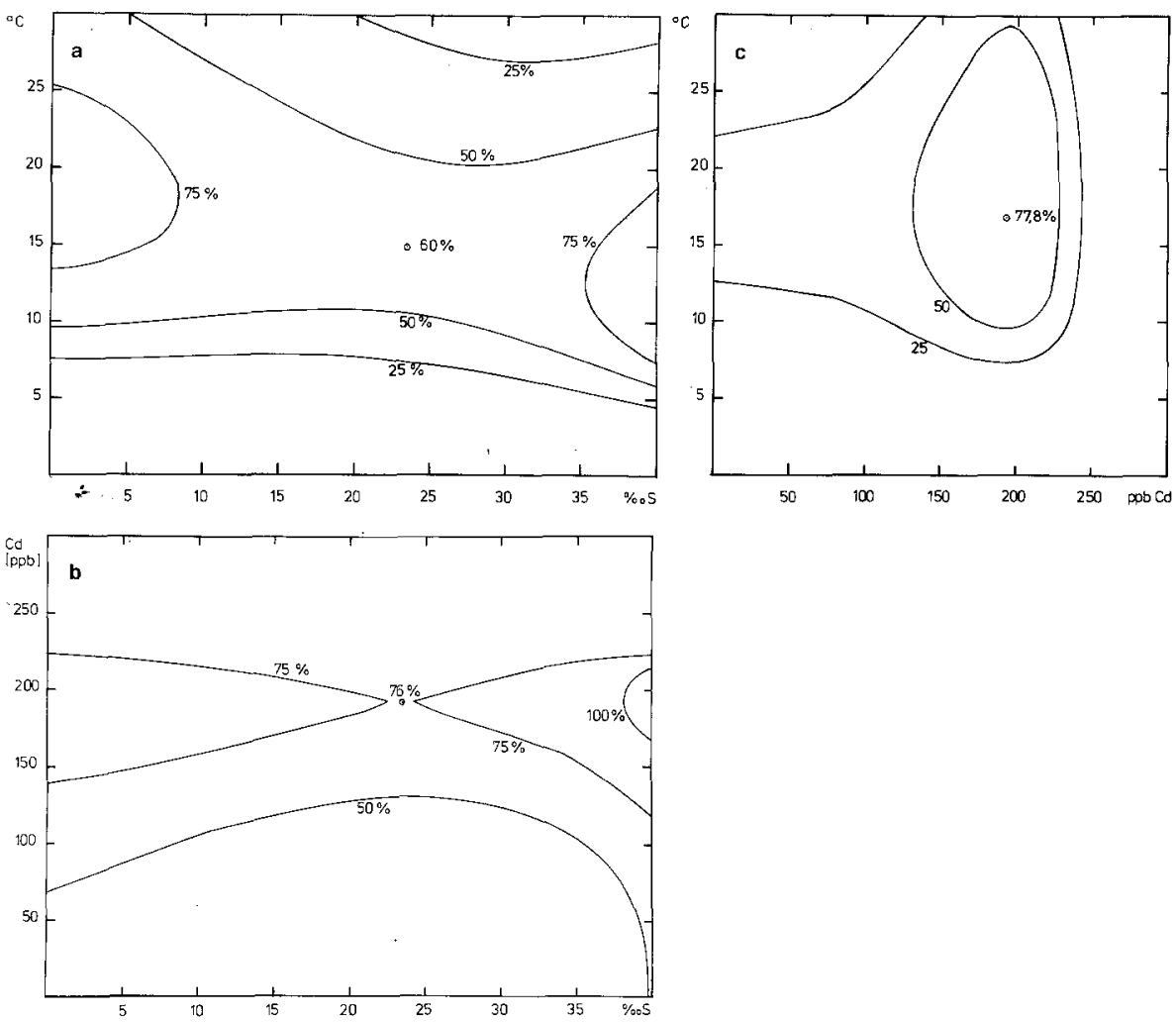

Abb. 6: Kumulierende Mortalität von Veliger-Larven 18 Tage nach Versuchsbeginn unter dem Einfluß von Temperatur, Salzgehalt und Cadmium, (a) T-S-Abhängigkeit bei $150 \mathrm{ppb} C \mathrm{~d}$ im Medium, (b) $\mathrm{Cd}$-S-Abhängigkeit bei $15^{\circ} \mathrm{C}$, (c) T-Cd-Abhängigkeit bei $21 \% \mathrm{~S}$ 
Unter dem andauernden Einfluß des Cadmiums erhöhen sich die kumulierenden Mortalitätsraten bis zu einem "Sättigungswert", der in verschiedenen Bereichen des Faktorenraums zu unterschiedlichen Zeitpunkten erreicht wird und aus methodischen Gründen in der Folgezeit wieder abzunehmen scheint. Diese beiden Effekte führen in den kommenden acht Versuchstagen zu Veränderungen in der Form des Reaktionskörpers, die sich nur mit der Kenntnis der Vorgeschichte der Mortalitätsraten erklären lassen.

18 Tage nach Versuchsbeginn liegt das Zentrum der Reaktionfläche ohne Cd-Zusatz mit $31 \%$ im Koordinatenpunkt $14{ }^{\circ} \mathrm{C}$ und $23 \%$ S. Es erfolgte demnach eine deutliche Anpassung an niedrige Salzgehalte. Das Zentrum des Reaktionskörpers (Abb. 6) mit einer Mortalitätsrate von $76 \%$ bei $194 \mathrm{ppb} \mathrm{Cd}^{2+}$ befindet sich im vergleichbaren Koordinatenpunkt von $14{ }^{\circ} \mathrm{C}$ und $20 \% \mathrm{~S}$. Die bis zum 10 . Tag beobachtete Wechselwirkung zwischen Cadmium und Salzgehalt einerseits sowie Temperatur andererseits wird prinzipiell nicht mehr verändert.

Die Dynamik des Wirkungszusammenhangs im Laufe der fortschreitenden Versuchsdauer geben am eindrucksvollsten die mathematischen Darstellungen wieder (Tab. 2). Vorgelegt werden die Parameter und Daten der linearen Analysen, die einsichtiger sind als die transformierten, nach denen die Reaktionsflächen erstellt wurden. Diese Art Regression erlaubt jedoch wegen ihrer geringen Flexibilität nur eine geringe Anpassung an die empirischen Daten. Dadurch treten gewisse Unterschiede in Form und Lage der Reaktionsflächen und in den Beträgen der Zentrumskoordinaten auf.

Von besonderem Interesse ist die zweite Versuchswoche (8. bis 15. Tag), in der eine deutliche Änderung des Wirkungszusammenhangs erfolgt, die sich im Verlauf der Beträge der Parameter der expliziten Form äußert. Der $b_{0}$-Wert und alle Parameter der linearen Effekte $\left(b_{1}-b_{3}\right)$ ändern in diesem Zeitraum ihr Vorzeichen, nachdem sie ein Minimum durchlaufen haben. Die Parameter der quadratischen $\left(b_{11}-b_{33}\right)$ - und intermediären $\left(b_{12}-b_{23}\right)$-Effekte erreichen in diesem Zeitraum zumeist ihre maximalen Absolutbeträge. Uber die relative Veränderung der Einflußstärke der einzelnen Faktoren (in Relation zu den Einflüssen der anderen Faktoren) geben die Absolutbeträge der Parameter der kanonischen Form Auskunft. Die Temperatur $\left(\mathrm{X}_{1}\right)$ nimmt während des gesamten Versuchszeitraums (bis zum 18. Tag) an Einfluß zu. Der Salzgehalt $\left(\mathrm{X}_{2}\right)$ und das Cadmium $\left(X_{3}\right)$ erreichen nach etwa einer Woche (8. Tag und 10. Tag) ihre maximale Wirkung und verlieren danach allmählich an Einfluß. Der zunehmende Einfluß des Cadmiums wird an der Konzentrationsabnahme seiner Zentrumskoordinate deutlich. Um eine geringe Mortalitätsrate aufrechtzuhalten, müssen die $\mathrm{Cd}$-Konzentrationen zunehmend vermindert werden. Nach Erreichen des "Sättigungszustandes" werden die Versuchsdaten mathematisch auf andere Weise interpretiert, was sich in einer deutlichen Veränderung der Zentrumskoordinaten und der Form und Lage der Reaktionsfläche ausdrückt.

\section{Wachstum der Veligerlarven}

Die Abhängigkeit des Wachstums der Larven von Mytilus edulis von unterschiedlichen T-S-Cd-Kombinationen verläuft übersichtlicher und klarer als die der kumulierenden Mortalität. Dargestellt werden die Reaktionsflächen der lineraren Regressionsanalysen, die in den meisten Fällen eine bessere Approximation liefern als die nichtlinearen. 
Tabelle 2

Parameter und charakteristische Daten der linearen Regressionen über die kumulierenden Mortalitä verschiedene Zeitpunkte nach Versuchsbeginn $\cdot b_{11}=$ Parameter der expliziten, linearen Regression, Änderung der Mortalität entlang der Achsen der Reaktionsfläche, $\mathrm{Y}=$ Mortalitätsrate, Winkelangabe $=$ Drehung der Achsen der Reaktionsfläche zu den Achsen des Faktorenfeldes, Zentrum aus, Zentrum = Minimum, -: Abfallen der Reaktionsfläche vom Zentrum aus,

\begin{tabular}{|lccccccccc}
\hline \multicolumn{1}{c}{$b_{0}$} & $b_{1}$ & $b_{2}$ & $b_{3}$ & $b_{11}$ & $b_{22}$ & $b_{33}$ & $b_{12}$ & $b_{13}$ & $b_{23}$ \\
\hline 3,3 Tage & & & & & & & & & \\
39,32 & $-3,07$ & $-0,504$ & $-0,038$ & 0,112 & $-0,010$ & $-0,00015$ & $-0,02$ & 0,0043 & 0,0023 \\
5,7 Tage & & & & & & & & & \\
63,96 & $-5,20$ & $-0,74$ & $-0,205$ & 0,173 & 0,013 & $(-0,00022)$ & $-0,025$ & 0,017 & 0,0034 \\
8 Tage & & & & & & & & & \\
83,89 & $-7,486$ & $-0,991$ & $-0,224$ & 0,314 & 0,039 & $\left(7,6 \times 10^{-6}\right)$ & $-0,074$ & 0,016 & 0,0031 \\
10,3 Tage & & & & & & & & & \\
68,37 & $-4,703$ & $-1,083$ & $-0,192$ & 0,244 & 0,073 & $(0,00021)$ & $-0,139$ & 0,022 & 0,0017 \\
12,7 Tage & & & & & & & & & \\
8,481 & 0,628 & 0,165 & 0,053 & 0,088 & 0,074 & $-0,00038$ & $-0,175$ & 0,017 & $-0,0037$ \\
15 Tage & & & & & & & & & \\
$-41,65$ & 5,341 & 1,436 & 0,214 & $-0,091$ & 0,043 & $-0,00069$ & $-0,158$ & 0,016 & $-0,0057$ \\
18 Tage & & & & & & & & & \\
$-34,16$ & 6,329 & $-0,454$ & 0,286 & $-0,111$ & 0,059 & $-0,00058$ & $-0,125$ & 0,009 & $-0,0026$ \\
21 Tage & & & & & & & & & \\
$-36,64$ & 6,331 & $-1,458$ & 0,563 & $(-0,051)$ & 0,0696 & $-0,00077$ & $-0,098$ & $-0,0054$ & $-0,0029$ \\
& & & & & & & & & \\
\hline
\end{tabular}

Die Einflüsse der Faktoren auf das Wachstum sind schwächer und nehmen langsamer zu als bei der Entwicklung und der Mortalität. Nach sechs Versuchstagen treten die wachstumsmodifizierenden Wirkungen nur schwach in weit auseinandergezogenen Isolinien in Erscheinung (Abb. 7), die einen schwach gekrümmten Reaktionskörper beschreiben, dessen Zentrum außerhalb der beobachteten Faktorenkombinationen liegt. Erst am 10. Tag stabilisiert sich die Abhängigkeit und bleibt bis auf eine Zunahme der Kapazität bis zum Ende des Versuchs konstant (Tab. 3).

Die Wirkungen der Faktoren unterscheiden sich deutlich von denen auf die Mortalität. Im Medium ohne Cadmiumzusatz tritt das Wachstumsoptimum bei $22{ }^{\circ} \mathrm{C}$ und $32 \% \mathrm{~S}$ auf und liegt damit bei einer höheren Temperatur und einem höheren Salzgehalt als das Mortalitätsminimum. Es erfolgt keine Verlagerung des Salzgehaltsoptimum, und suboptimale Konzentrationen hemmen die Wachstumsrate sțärker als die Überlebensrate. Auf Grund einer wechselseitigen Beeinflussung von Temperatur und Salzgehalt auf das Wachstum kann der durch Verminderung eines Faktors herabgesetzten Wachstumsrate durch 
Tabelle 2

ten von Veliger-Larven in Abhängigkeit von Temperatur, Salzgehalt und Cadmiumkonzentration für in Klammern Glieder ohne Signifikanz; die Parameter der kanonischen Gleichung sind ein Maß für die $\mathrm{X}_{1}=$ Temperatur, $\mathrm{X}_{2}=$ Salzgehalt, $\mathrm{X}_{3}=$ Cadmiumkonzentration; $\mathrm{Y}_{\mathrm{s}}, \mathrm{X}_{\mathrm{is}}=$ Zentrumskoordinaten: darunter Vorzeichenangabe über die Form der Reaktionsfläche, + : Ansteigen der Fläche vom Zentrum = Maximum entlang der entsprechenden Achse

\begin{tabular}{|c|c|c|c|c|c|c|c|}
\hline Kanonische Gleichung & $\underset{(\%)}{Y_{s}}$ & $\underset{\left({ }^{\circ} \mathrm{C}\right)}{ }$ & $\begin{array}{c}\mathrm{X}_{2 s} \\
(\% \infty \mathrm{S}) \\
\end{array}$ & $\begin{array}{c}\mathrm{X}_{3 \mathrm{~s}} \\
(\mathrm{ppb} \mathrm{Cd})\end{array}$ & $\begin{array}{r}F \\
X_{1} / X_{2} \\
\end{array}$ & $\begin{array}{l}\mathrm{rm} \text { und } \\
\mathrm{X}_{1} / \mathrm{X}_{3}\end{array}$ & $\stackrel{\text { age }}{\mathrm{X}_{2}} / \mathrm{X}_{3}$ \\
\hline $\begin{aligned} \mathrm{Y}-11,21= & -0,00048 \mathrm{X}_{1}{ }^{2} \\
& +0,113 \mathrm{X}_{2}^{2} \\
& +0,0093 \mathrm{X}_{3}{ }^{2}\end{aligned}$ & $\begin{array}{l}11,21 \\
19,2\end{array}$ & $\begin{array}{l}13 \\
17,5\end{array}$ & $\begin{array}{l}21,9 \\
42,1\end{array}$ & $\begin{array}{c}137,8 \\
0\end{array}$ & $\begin{array}{l}5,5^{\circ} \\
+/+\end{array}$ & $\begin{array}{l}89^{\circ} \\
-1+\end{array}$ & $\begin{array}{l}84^{\circ} \\
-1+\end{array}$ \\
\hline $\begin{aligned} \mathrm{Y}-14,74= & +0,1739 \mathrm{X}_{1}{ }^{2} \\
& -0,0010 \mathrm{X}_{2}{ }^{2} \\
& +0,0123 \mathrm{X}_{3}{ }^{2}\end{aligned}$ & $\begin{array}{c}14,74 \\
1,1\end{array}$ & $\begin{array}{l}10,9 \\
18,4\end{array}$ & $\begin{array}{l}22,9 \\
46,5\end{array}$ & $\begin{array}{c}122,5 \\
0\end{array}$ & $\begin{array}{l}4,4^{\circ} \\
+1+\end{array}$ & $\begin{array}{l}87^{\circ} \\
-1+\end{array}$ & $\begin{array}{l}83^{\circ} \\
-1+\end{array}$ \\
\hline $\begin{aligned} \mathrm{Y}-20,95= & -0,00037 \mathrm{X}_{1}{ }^{2} \\
& +0,319 \mathrm{X}_{2}{ }^{2} \\
& +0,0346 \mathrm{X}_{3}{ }^{2}\end{aligned}$ & $\begin{array}{l}21 \\
14,3\end{array}$ & $\begin{array}{l}10,5 \\
15\end{array}$ & $\begin{array}{l}17,2 \\
26,7\end{array}$ & $\begin{array}{c}133,7 \\
0\end{array}$ & $\begin{array}{l}7,5^{\circ} \\
+1+\end{array}$ & $\begin{array}{l}88,5^{\circ} \\
-1+\end{array}$ & $\begin{array}{l}88^{\circ} \\
+1-\end{array}$ \\
\hline $\begin{aligned} \mathrm{Y}-28,05= & -0,00097 \mathrm{X}_{1}^{2} \\
& +0,2693 \mathrm{X}_{2}^{2} \\
& +0,0487 \mathrm{X}_{3}^{2}\end{aligned}$ & $\begin{array}{l}28,1 \\
18,3\end{array}$ & $\begin{array}{r}9,6 \\
16,1\end{array}$ & $\begin{array}{l}15,3 \\
22,7\end{array}$ & $\begin{array}{c}99,7 \\
0\end{array}$ & $\begin{array}{l}19,5^{\circ} \\
+/+\end{array}$ & $\begin{array}{l}87,5^{\circ} \\
-1+\end{array}$ & $\begin{array}{l}89^{\circ} \\
-1+\end{array}$ \\
\hline $\begin{aligned} Y-17,36= & -0,00961 X_{1}{ }^{2} \\
& +0,1689 X_{2}^{2} \\
& +0,0017 X_{3}^{2}\end{aligned}$ & $\begin{array}{l}17,4 \\
19,4\end{array}$ & $\begin{array}{l}10,1 \\
26,8\end{array}$ & $\begin{array}{l}15,3 \\
30,5\end{array}$ & $\begin{array}{c}87,3 \\
0\end{array}$ & $\begin{array}{l}42,7^{\circ} \\
+1-\end{array}$ & $\begin{array}{l}84,4^{\circ} \\
-1+\end{array}$ & $\begin{array}{l}1,4^{\circ} \\
+/-\end{array}$ \\
\hline $\begin{aligned} Y-64,35= & -0,1274 X_{1}^{2} \\
& +0,0803 X_{2}^{2} \\
& -0,00083 X_{3}^{2}\end{aligned}$ & $\begin{array}{l}64,4 \\
14,1\end{array}$ & $\begin{array}{l}20,2 \\
17,1\end{array}$ & $\begin{array}{l}36,1 \\
14,3\end{array}$ & $\begin{array}{c}244,9 \\
0\end{array}$ & $\begin{array}{l}65,2^{\circ} \\
+1-\end{array}$ & $\begin{array}{l}5,2^{\circ} \\
-1+\end{array}$ & $\begin{array}{l}3,7^{\circ} \\
+1-\end{array}$ \\
\hline $\begin{aligned} Y-78,78= & -0,1316 X_{1}^{2} \\
& +0,0796 X_{2}^{2} \\
& -0,00055 X_{3}^{2}\end{aligned}$ & $\begin{array}{l}78,8 \\
13,4\end{array}$ & $\begin{array}{l}22,2 \\
16,6\end{array}$ & $\begin{array}{l}35,1 \\
21,2\end{array}$ & $\begin{array}{r}343 \\
0\end{array}$ & $\begin{array}{l}71,9^{\circ} \\
+1-\end{array}$ & $\begin{array}{l}2,3^{\circ} \\
-1-\end{array}$ & $\begin{array}{l}1,2^{\circ} \\
+1-\end{array}$ \\
\hline $\begin{aligned} Y-73,45= & +0,08697 X_{1}^{2} \\
& -0,0687 X_{2}^{2} \\
& -0,000637 X_{3}^{2}\end{aligned}$ & $\begin{array}{l}73,5 \\
37,9\end{array}$ & $\begin{array}{l}20,7 \\
31\end{array}$ & $\begin{array}{l}30,0 \\
32\end{array}$ & $\begin{array}{c}235,9 \\
0\end{array}$ & $\begin{array}{l}70,5^{\circ} \\
+1-\end{array}$ & $\begin{array}{l}87^{\circ} \\
-1-\end{array}$ & $\begin{array}{l}1,2^{\circ} \\
+1-\end{array}$ \\
\hline
\end{tabular}

eine gleichzeitige Verminderung des anderen Faktors entgegengewirkt werden. Diese mit einem negativen Vorzeichen versehene Wechselwirkung (Drehung der Reaktionsfläche entgegen dem Uhrzeigersinn) zeigt einen umgekehrten Effekt (Abb. 8a) als bei der Entwicklung der Larven. Der Einfluß des Cadmiums auf das salzgehaltsabhängige Wachstum (Abb. 8b) entspricht etwa dem auf die Mortalität. Zunehmende Cd-Konzentrationen rücken das Wachstumsoptimum in einen geringeren Salzgehaltsbereich. Zwischen dem Schwermetall und der Temperatur wird keine Wechselwirkung beobachtet. Die Stärke des Schwermetalleinflusses erscheint im optimalen Salz- und Temperaturbereich am ausgeprägtesten (Abb. $8 \mathrm{~b}, \mathrm{c}$ ).

Bis zum 18. Tag nach Versuchsbeginn wird das Wachstum im Bereich des optimalen Salzgehalts oder der Optimaltemperatur durch $150 \mathrm{ppb} \mathrm{Cd}^{2+}$ im Medium um etwa $25 \%$ gehemmt. Die Stärke dieser Wirkung ist vergleichbar mit einer Verminderung des Salzgehalts um $15 \% \mathrm{~S}$ oder einer Temperaturänderung um etwa $15{ }^{\circ} \mathrm{C}$, ausgehend vom Optimalwert. 
Tabelle 3

Parameter und charakteristische Daten der linearen Regressionen über das Veliger-Wachstum in nach Versuchsbeginn (siehe Legende in Tab. 2)

\begin{tabular}{|c|c|c|c|c|c|c|c|c|c|}
\hline$b_{o}$ & $b_{1}$ & $b_{2}$ & $b_{3}$ & $\mathrm{~b}_{11}$ & $\mathbf{b}_{22}$ & $b_{33}$ & $\mathrm{~b}_{12}$ & $\mathrm{~b}_{13}$ & $b_{23}$ \\
\hline $\begin{array}{l}\text { 6. Tag } \\
85,96\end{array}$ & 0,884 & 1,873 & $-0,0007$ & $(-0,016)$ & $-0,050$ & $(0,00016)$ & 0,0799 & $(-0,00162)$ & $-0,0040$ \\
\hline $\begin{array}{l}\text { 8. Tag } \\
56,81\end{array}$ & 4,368 & 2,498 & 0,0384 & $-0,136$ & $-0,0687$ & $\left(-0,94 \times 10^{-4}\right)$ & 0,0987 & $(-0,00112)$ & $-0,0040$ \\
\hline $\begin{array}{l}\text { 10,3. Tag } \\
22,06\end{array}$ & 8,864 & 3,667 & $-0,015$ & $-0,293$ & $-0,101$ & $\left(0,695 \times 10^{-4}\right)$ & 0,125 & $(-0,0014)$ & $-0,0045$ \\
\hline $\begin{array}{l}\text { 12,7. Tag } \\
2,97\end{array}$ & 10,97 & 4,13 & 0,0014 & $-0,35$ & $-0,107$ & $(0,00027)$ & 0,137 & $(-0,0044)$ & $-0,0065$ \\
\hline $\begin{array}{l}\text { 15. Tag } \\
10,58\end{array}$ & 8,64 & 5,54 & $-0,120$ & $-0,268$ & $-0,128$ & $(0,00062)$ & 0,123 & $(0,00050)$ & $-0,0085$ \\
\hline $\begin{array}{l}\text { 18. Tag } \\
-5,324\end{array}$ & 9,312 & 6,458 & $-0,0456$ & $-0,291$ & $-0,148$ & $(0,00038)$ & 0,1355 & $(-0,00116)$ & 0,00876 \\
\hline $\begin{array}{l}\text { 21. Tag } \\
-13,05\end{array}$ & 8,72 & 7,70 & 0,014 & $-0,275$ & $-0,184$ & $\left(0,68 \times 10^{-4}\right)$ & 0,149 & $(-0,0004)$ & $-0,00825$ \\
\hline
\end{tabular}

Die erwähnte konstante Form und Lage des Reaktionskörpers vom 10. Versuchstag an kommt in den mathematischen Analysen (Tab. 3) über die nur unwesentlich schwankenden, entsprechenden Daten sowie über die Zentrumskoordinaten ohne Cd-Zusatz zum Ausdruck. Der ansteigenden Kapazität und den zunehmenden Einflüssen von Temperatur und Salzgehalt entspricht die kontinuierliche Veränderung der Werte von $b_{0}$ und $b_{1}, b_{2}, b_{11}$, $b_{22}$ für Temperatur und Salzgehalt. Die auffälligen Schwankungen der Parameterwerte des linearen $\mathrm{Cd}$-Gliedes $\left(\mathrm{b}_{3}\right)$ - und im Gefolge ebenfalls die der Zentrumskoordinaten und die der kanonischen Gleichung - sind nur mit einer selektiven Mortalität bestimmter Größenklassen zu erklären, die in zeitlich aufeinanderfolgenden Wellen erfolgt. Die Größenverteilungen einiger Populationen zeigen zeitweise einen zweigipfligen Verlauf, der später in einen eingipfligen übergeht. Es bleibt jedoch einer weiteren Datenanalyse überlassen, festzustellen, ob der klein- oder der großwüchsige Veligeranteil einer höheren Mortalitätsrate unterlag.

\section{DISKUSSION}

Uber die Temperatur- und Salzgehaltsansprüche der Larven von Mytilus edulis liegen detaillierte Untersuchungen von Bayne (1965) vor. Die von ihm bestimmten oberen und unteren Temperaturgrenzen von $5{ }^{\circ} \mathrm{C}$ und $20-22^{\circ} \mathrm{C}$, innerhalb derer eine Befruchtung und 
Abhängigkeit von Temperatur, Salzgehalt und Cadmiumkonzentration für verschiedene Zeitpunkte

\begin{tabular}{|c|c|c|c|c|c|c|c|c|}
\hline Kanonisch & ae Gleichung & $\begin{array}{l}Y_{s} \\
(\mu)\end{array}$ & $\begin{array}{l}\mathrm{X}_{1 \mathrm{~s}} \\
\left({ }^{\circ} \mathrm{C}\right) \\
\end{array}$ & $\begin{array}{c}\mathrm{X}_{2 \mathrm{~s}} \\
(\% 0 \mathrm{~S})\end{array}$ & $\underset{\left(\mathrm{ppb} \mathrm{Cd^{2+ }}\right)}{\mathrm{X}_{3 \mathrm{~s}}}$ & $\mathrm{X}_{1} / \mathrm{X}_{2}^{\mathrm{F}}$ & $\mathrm{m}_{\mathrm{X}}$ und & $\begin{array}{l}\text { age } \\
\mathrm{X}_{2} / \mathrm{X}_{3}\end{array}$ \\
\hline$Y-180,84$ & $\begin{aligned}= & -0,076 \mathrm{X}_{1}^{2} \\
& -0,00012 \mathrm{X}_{2}^{2} \mathrm{~W} \\
& +0,011 \mathrm{X}_{3}^{2} \mathrm{~W}\end{aligned}$ & $\begin{array}{l}180,8 \\
147,2\end{array}$ & $\begin{array}{r}102,3 \\
74,6\end{array}$ & $\begin{array}{l}43,46 \\
40,9\end{array}$ & $\begin{array}{r}1166 \\
0\end{array}$ & $\begin{array}{l}56^{\circ} \\
-1+\end{array}$ & $\begin{array}{l}87^{\circ} \\
+1-\end{array}$ & $\begin{array}{l}87^{\circ} \\
+/-\end{array}$ \\
\hline$Y-1067,2$ & $\begin{aligned}= & -0,1619 \mathrm{X}_{1}^{2} \\
& +0,501 \times 10^{-5} \mathrm{X}_{2}^{2} \\
& -0,043 \mathrm{X}_{3}^{2}\end{aligned}$ & $\begin{array}{c}1067 \\
174,1\end{array}$ & $\begin{array}{r}283,4 \\
30,7\end{array}$ & $\begin{array}{r}646,2 \\
40,2\end{array}$ & $\begin{array}{c}157 \times 10^{4} \\
0\end{array}$ & $\begin{array}{l}28^{\circ} \\
-1-\end{array}$ & $\begin{array}{l}90^{\circ} \\
-1-\end{array}$ & $\begin{array}{l}88^{\circ} \\
-1-\end{array}$ \\
\hline$Y-112,24$ & $\begin{aligned}= & -0,082 \mathrm{X}_{1}^{2} \\
& +0,00014 \mathrm{X}_{2}^{2} \\
& -0,311 \mathrm{X}_{3}^{2}\end{aligned}$ & $\begin{array}{l}112,2 \\
178,0\end{array}$ & $\begin{array}{l}16,2 \\
22\end{array}$ & $\begin{array}{l}12,8 \\
31,9\end{array}$ & $\begin{array}{c}688,8 \\
0\end{array}$ & $\begin{array}{l}16^{\circ} \\
-1-\end{array}$ & $\begin{array}{l}90^{\circ} \\
+1-\end{array}$ & $\begin{array}{l}89^{\circ} \\
+1-\end{array}$ \\
\hline$Y-135,6$ & $\begin{aligned}= & +0,00043 \mathrm{X}_{1}^{2} \\
& -0,369 \mathrm{X}_{2}^{2} \\
& -0,089 \mathrm{X}_{3}^{2}\end{aligned}$ & $\begin{array}{l}135,6 \\
193,2\end{array}$ & $\begin{array}{l}17,0 \\
22,1\end{array}$ & $\begin{array}{l}18,9 \\
33,4\end{array}$ & $\begin{array}{c}366,5 \\
0\end{array}$ & $\begin{array}{l}14,6^{\circ} \\
-/-\end{array}$ & $\begin{array}{l}90^{\circ} \\
+1-\end{array}$ & $\begin{array}{l}88^{\circ} \\
+1-\end{array}$ \\
\hline$Y-156$ & $\begin{aligned}= & -0,291 \mathrm{X}_{1}^{2} \\
& +0,00087 \mathrm{X}_{2}^{2} \\
& -0,105 \mathrm{X}_{3}^{2}\end{aligned}$ & $\begin{array}{l}156 \\
204,8\end{array}$ & $\begin{array}{l}21,9 \\
23,7\end{array}$ & $\begin{array}{l}23,8 \\
33,1\end{array}$ & $\begin{array}{c}250,8 \\
0\end{array}$ & $\begin{array}{l}20,7^{\circ} \\
-1-\end{array}$ & $\begin{array}{l}0^{\circ} \\
-1+\end{array}$ & $\begin{array}{l}88^{\circ} \\
+1-\end{array}$ \\
\hline $\mathrm{Y}-149,9$ & $\begin{aligned}= & -0,318 \mathrm{X}_{1}^{2} \\
& +0,00054 \mathrm{X}_{2}^{2} \\
& -0,121 \mathrm{X}_{3}^{2}\end{aligned}$ & $\begin{array}{l}149,9 \\
209,6\end{array}$ & $\begin{array}{l}20,3 \\
23,6\end{array}$ & $\begin{array}{l}21,2 \\
32,5\end{array}$ & $\begin{array}{c}333,9 \\
0\end{array}$ & $\begin{array}{l}21,8^{\circ} \\
-1-\end{array}$ & $\begin{array}{l}90^{\circ} \\
+1-\end{array}$ & $\begin{array}{l}88^{\circ} \\
+1-\end{array}$ \\
\hline $\mathrm{Y}-122,4$ & $\begin{aligned}= & -0,317 \mathrm{X}_{1}^{2} \\
& +0,00017 \mathrm{X}_{2}^{2} \\
& -0,142 \mathrm{X}_{3}^{2}\end{aligned}$ & $\begin{array}{l}122,4 \\
211,1\end{array}$ & $\begin{array}{l}18,8 \\
24,3\end{array}$ & $\begin{array}{l}12,6 \\
30,7\end{array}$ & $\begin{array}{c}713,4 \\
0\end{array}$ & $\begin{array}{l}29,4^{\circ} \\
-1-\end{array}$ & $\begin{array}{l}90^{\circ} \\
+1-\end{array}$ & $\begin{array}{l}89^{\circ} \\
+1-\end{array}$ \\
\hline
\end{tabular}

Schalenbildung stattfindet, stimmen gut mit den vorliegenden und vergleichbaren Daten über die Entwicklung der befruchteten Eier bis zu den Trochophora-Larven (Abb. 1 und 2) überein. Vergleichbar ist auch die von ihm angegebene Salzgehaltsspanne von 15 bis $40 \% \mathrm{~S}$, in der eine Befruchtung erfolgt (vgl. Abb. 1). Für die vom Salzgehalt abhängige weitere Entwicklung spielt nach Bayne (1965) auch der Herkunftssalzgehalt der Eltern eine Rolle. Er unterscheidet zwischen Nachkommen mariner Eltern, bei denen die Entwicklung zxischen 30 und $40 \% \mathrm{~S}$ erfolgt und solchen von Brackwassermuttertieren, bei denen sie auf den Bereich von 11-30\% S verschoben sein kann. Die letzteren Werte decken sich praktisch mit denen der vorliegenden Arbeit. Die eintretende Plastizitätsänderung zwischen dem Zeitpunkt der Befruchtung und der Entwicklung zur Veliger-Larve kommt in der sich verändernden Temperatur-Salzgehaltsabhängigkeit zum Ausdruck, die zudem noch von einer andersartigen Abhängigkeit der Mortalitätsraten beeinflußt wird (Abb. 3 und 4).

Für das Wachstum (vgl. Abb. 8) kann in Ubereinstimmung mit Bayne eine Temperatur von 5 bis $10^{\circ} \mathrm{C}$ als untere Begrenzung angegeben werden, auch die modifizierenden Wirkungen von Temperatur und Salzgehalt auf das Wachstum, die mit ansteigenden Temperaturen den optimalen Salzgehaltswert erhöhen $\left(13{ }^{\circ} \mathrm{C}\right.$ : $15,5-26 \% \mathrm{~S} ; 16^{\circ} \mathrm{C}$ : 20-30\% S) stimmen mit Bayne überein. Unterschiedlich sind jedoch die Temperaturoptima: $15^{\circ} \mathrm{C}$ (bei 20-30\% $\%$, Bayne, 1965) und 22-24 ${ }^{\circ} \mathrm{C}$ (bei 31-33\% $\%$, vgl. Tab. 3). 

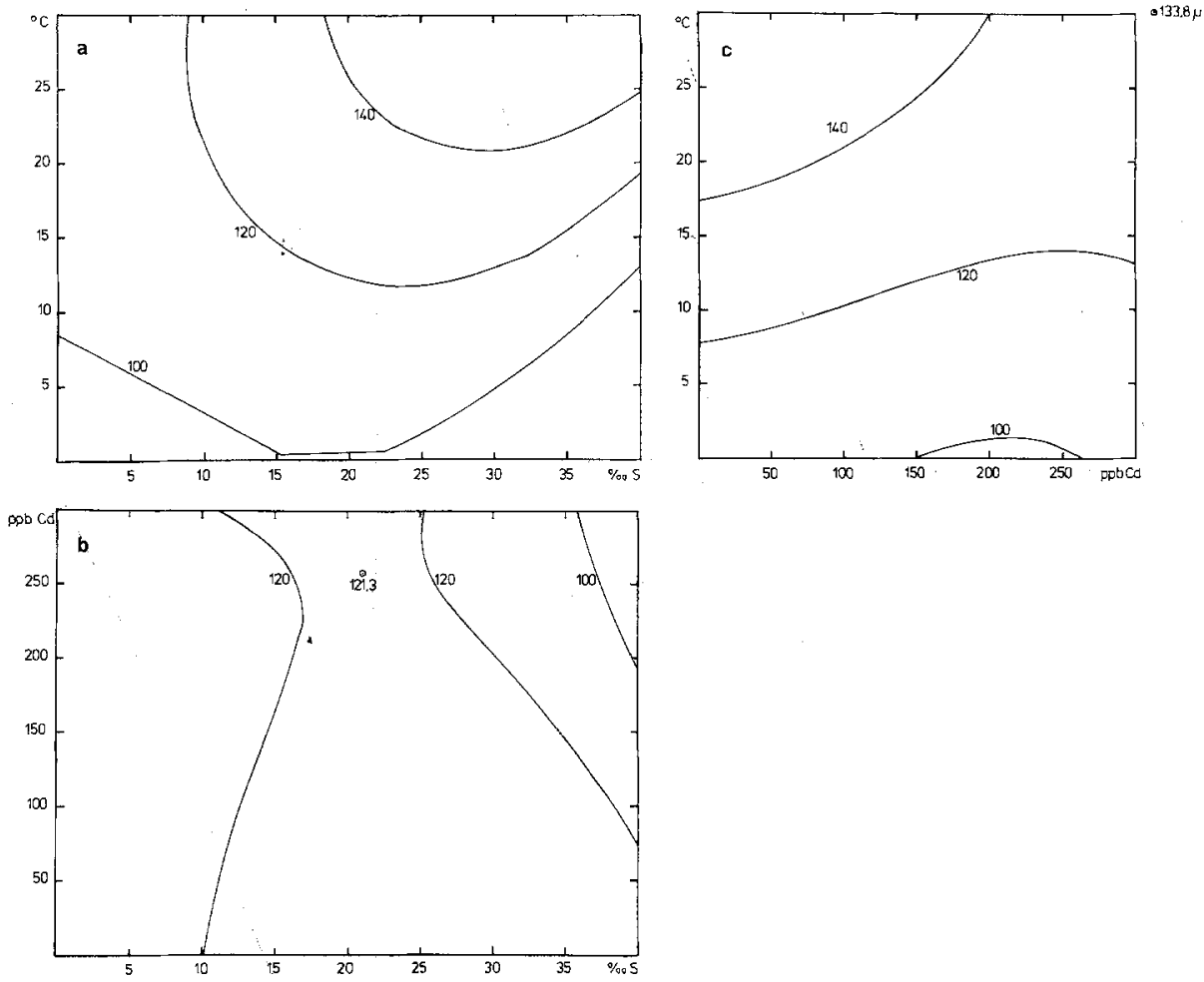

Abb. 7: Wachstum von Veligerlarven unter dem Einfluß von Temperatur, Salzgehalt und CadmiumKonzentration (6 Tage nach Versuchsbeginn), (a) T-S-Abhängigkeit bei $150 \mathrm{ppb} \mathrm{Cd}$ im Medium, (b) Cd-S-Abhängigkeit bei $15^{\circ} \mathrm{C}$, (c) T-Cd-Abhängigkeit bei $21 \% \mathrm{~S}$

Nach Brenko \& Calabrese (1969) liegt das Wachstumsoptimum der Larven von Mytilus edulis um $20^{\circ} \mathrm{C}$ und $30 \% \mathrm{~S}$, bei ähnlichen Temperatur-Salzgehaltskombinationen also, wie nach den Ergebnissen dieser Arbeit. Möglicherweise wird das Temperaturoptimum noch durch weitere, nicht kontrollierte Faktoren beeinflußt.

Die optimalen Salzgehaltswerte für die Entwicklung einer Trochophora- und für das Wachstum und die Úberlebensrate einer Veligerpopulation liegen jedoch im Bereich voll mariner Bedingungen. Sie zeigen bis zum Alter der Larven von etwa 4 Wochen - abgesehen von der Uberlebensrate (vgl. Tab. 2) - keine Tendenz einer Verlagerung zu den Salzkonzentrationen hin, die die Tiere am Standort in der Ostsee vorfinden. Diese Diskrepanz wird in beiden Entwicklungsstadien auf unterschiedliche Weise kompensiert. Bei der nur eine kurze Zeitspanne existenten planktonischen Trochophora erscheint das Muster der temperatur-salzgehaltsabhängigen Entwicklung besonders für das Ertragen der kurzfristigen, saisonalen und vertikalen Faktorenschwankungen im Ostseewasser geeignet. Der Vertikalgradient der Temperatur/Salzgehaltsbedingungen im westlichen Teil der Ostsee verläuft dabei von niedrig/hoch (Tiefe) zu hoch/niedrig (Oberfläche) (vgl. Abb. 1). Die längerlebige Veligerlarve ist auf Grund der temperatur-salzgehaltsabhängigen Toleranzen von Wachstum und Uberlebensfähigkeit eher langfristigen und weiträumig horizontalen 

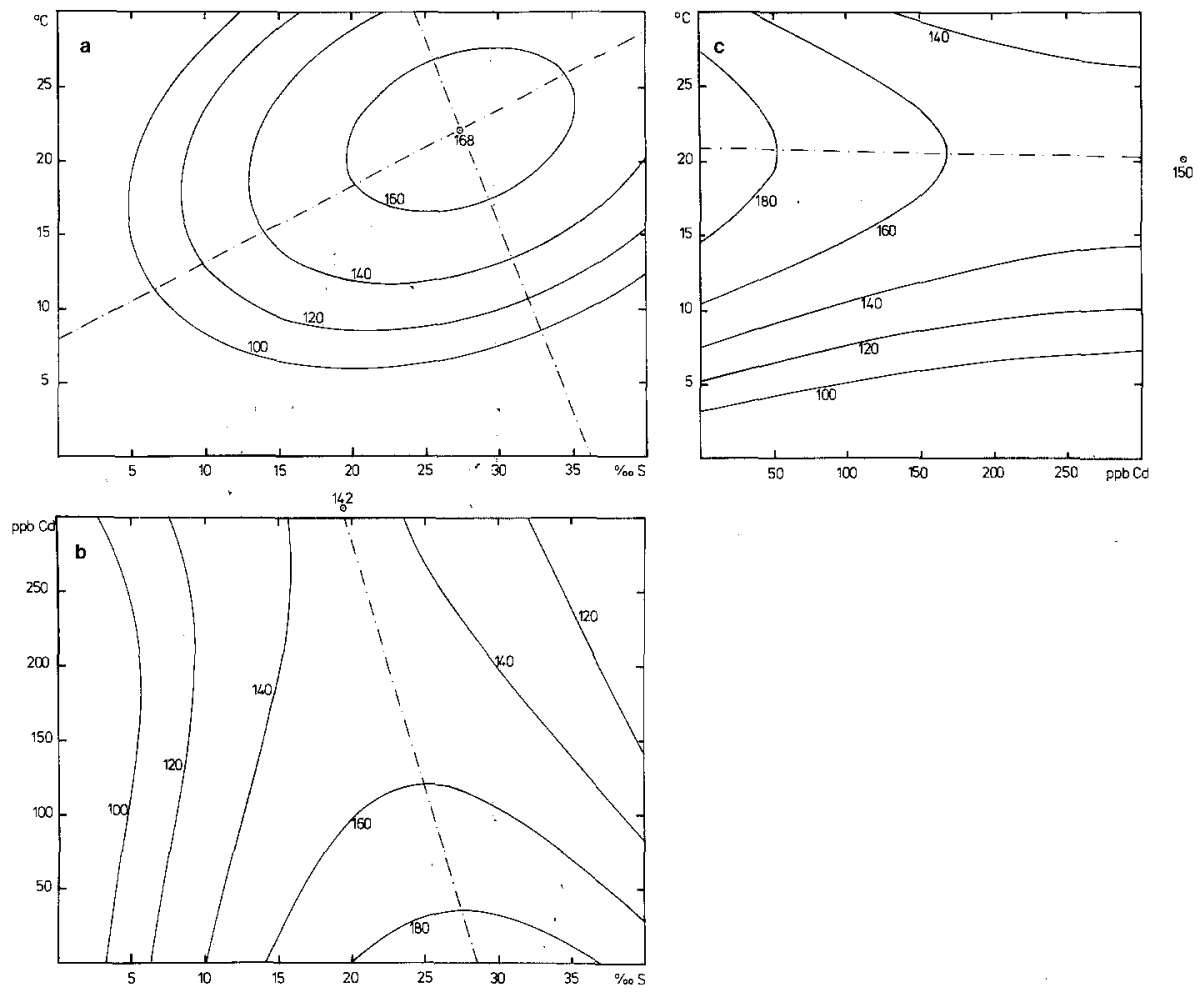

Abb. 8: Wachstum von Veligerlarven unter dem Einfluß von Temperatur, Salzgehalt und CadmiumKonzentration (18 Tage nach Versuchsbeginn), (a) T-S-Abhängigkeit für $150 \mathrm{ppb} \mathrm{Cd}$ im Medium, (b) Cd-S-Abhängigkeit bei $15^{\circ} \mathrm{C}$, (c) T-Cd-Abhängigkeit bei $21 \% \mathrm{~S}$

Faktorenschwankungen der Ostsee angepaßt, die mit zunehmender Jahreszeit und im OstWest-Gradienten der Tendenz niedrig/niedrig - hoch/hoch folgen.

Ungünstige Auswirkungen von Cadmium werden in dieser Untersuchung schon bei verhältnismäßig geringen Konzentrationen (ab etwa 50 ppb) deutlich. Pavicic \& Järvenpää (1974) registrierten dagegen an Larven von Mytilus galloprovincialis gesteigerte Mortalitätsraten erst im ppm-Bereich. Rosenberg \& Costlow (1976) allerdings beobachteten an Larven der Krabben Callinectes sapidus und $R(b)$ ithropanopeus harrisii ebenfalls meßbare Einflüsse bereits ab $50 \mathrm{ppb}$, während Brown \& Ahsanullah (1971) an Adulten von Artemia salina nach 10 Tagen einen $\mathrm{LD}_{50}$-Wert von $1 \mathrm{ppm}$ fanden. Verschiedene Entwicklungsstadien und Tierarten unterscheiden sich offensichtlich stark in ihrer Empfindlichkeit gegenüber Cadmium.

In der Larvalentwicklung von Mytilus edulis zeigt sich das Trochophorastadium als empfindlichster Entwicklungsabschnitt gegenüber Temperatur- und Salzgehaltseinwirkungen. Die hohen Mortalitätsraten in einem dreifaktoriellen Versuch mit Cadmiumzugaben sind möglicherweise auf einen stark schädigenden Einfluß des Schwermetalls zurückzuführen. Sichere Angaben über seinen Einfluß können erst für den Zeitraum des Ubergangs von der Trochophora- zur Veliger-Larve ermittelt werden. In diesem Entwicklungsabschnitt 

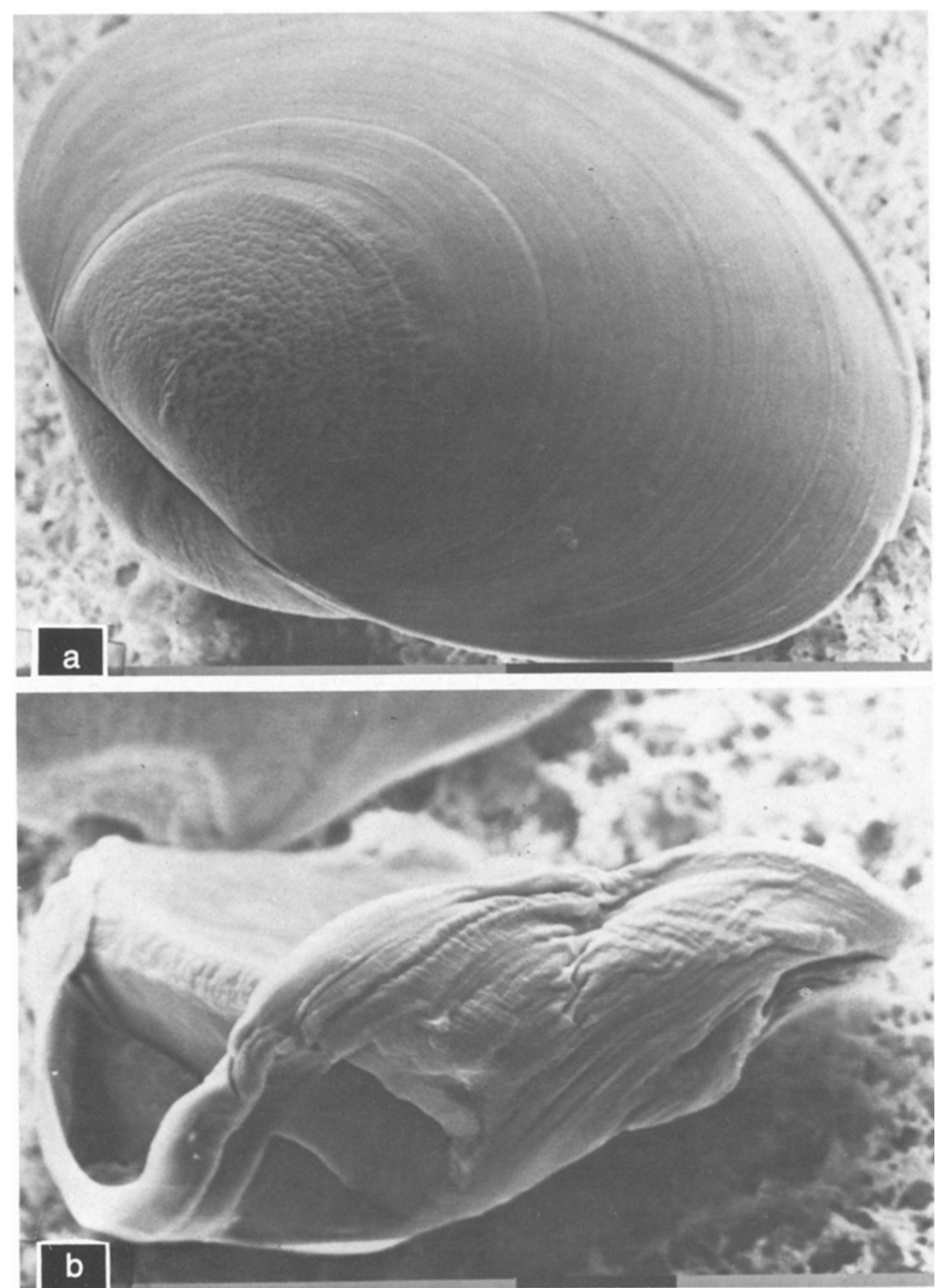

Abb. 9: Rasterelektronenmikroskopische Aufnahmen von Veligerlarven, oben normale Schalenform einer Larve $\left(15^{\circ} \mathrm{C} / 21 \% \mathrm{~S} / 3,8 \mathrm{ppb} \mathrm{Cd}^{2+}\right)$, unten mißgebildete Schale einer Larve $\left(15^{\circ} \mathrm{C} / 21 \% \mathrm{~S} /\right.$ $233 \mathrm{ppb} \mathrm{Cd}^{2+}$ ) 
der Beschalung der Larven liegt eine starke Wechselwirkung zwischen Salzgehalt und Schwermetall vor (Abb. 3): Mit zunehmender Cd-Konzentration wird das salzgehaltsabhängige Entwicklungsoptimum zu höheren Salzkonzentrationen hin verlagert (z. B. erfolgt bei Anstieg der Cd-Konzentration von 0 auf 50 ppb eine Verlagerung des Entwicklungsoptimums von $25 \%$ auf etwa $33 \% \mathrm{~S} ; 15{ }^{\circ} \mathrm{C}$ ). Bei der Größenordnung dieses Schwermetalleinflusses können bereits geringe $\mathrm{Cd}$-Konzentrationen zur Folge haben, daß sich der für die Entwicklung optimale Salzgehaltswert außerhalb der für die Ostseetiere zugänglichen Konzentrationen befindet. Eine im Prinzip vergleichbare Beziehung wurde von v. Westernhagen et al. (1974) bei der Cadmium-Kontamination von Heringseiern beobachtet und auf eine Wechselwirkung zwischen $\mathrm{Cd}^{2+}$ - und dem $\mathrm{Ca}^{2+}$-Gehalt des Meerwassers zurückgeführt (Kobayashi, 1971). Auch Mytilus edulis entwickelt in diesem Entwicklungsabschnitt einen erhöhten Calziumbedarf für den beginnenden Schalenbau. Einer Verlagerung des Salzgehaltsoptimums in salzreichere Wasser entspricht ein erhöhtes Calziumangebot, das den "Gegenspieler" Cadmium gewissermaßen kompensiert. Schon äußerlich läßt șich der Einfluß des Schwermetalls auf den Schalenbau erkennen (Abb. 9).

Es ist bemerkenswert, daß sich mit dem Ubergang zur Veligerlarve die CadmiumSalzgehaltswechselwirkung umkehrt, so daß nun mit steigenden Cadmiumkonzentrationen das Mortalitätsminimum und das Wachstumsoptimum zu niedrigeren Salzgehalten hin verlagert werden (Tab. 2 und 3). Mit dem Úbergang zur Veliger vollziehen sich offensichtlich Veränderungen in der Larve, die sich morphologisch am unterschiedlichen Schalenbau (Abb. 9) und physiologisch an der Ausbildung einer erhöhten Resistenz gegenüber niedrigen Salzgehalten zeigen (vgl. Abb. 1-3 mit Abb. 5-7). Diese Plastizitätsänderung verläuft im gleichen Sinne, wie die Änderung der Beziehungen zwischen Schwermetall und Salzgehalt.

Betrachtet man die Wirkung der drei Faktoren unter dem Gesichtspunkt der begrenzenden Faktoren (Blackman, 1905), so werden die dominierenden Einflüsse von beispielsweise Temperatur und Salzgehalt in ihren Grenzbereichen und der steigende Einfluß des Cadmiums zu den Temperatur- und Salzgehaltsoptima hin verständlich. Durch die gegenseitige Beeinflussung der Faktoren in ihrer Wirkung auf den Organismus wird dieses Prinzip modifiziert, was insbesondere zu Verlagerungen der Grenzbereiche, d.h. zu Verschiebungen der Toleranzgrenzen, führt.

Die vorliegenden Ergebnisse machen insbesondere deutlich, daß bereits unterhalb letaler Cd-Konzentrationen das feinabgestimmte Wechselspiel zwischen den Reaktionen der Larven von Mytilus edulis und den natürlichen Umweltbedingungen (hier auf Temperatur und Salzgehalt reduziert) durch ein Schwermetall wie Cadmium gestört wird. Da sich zudem in den ersten vier Wochen der Larvalentwicklung die Abhängigkeiten von Temperatur und Salzgehalt ändern und diese Abhängigkeiten zu verschiedenen Zeitpunkten in unterschiedlicher Weise vom Cadmium beeinflußt werden, kann davon ausgegangen werden, daß sich die hier im einzelnen wiedergegebenen, negativen Auswirkungen aufsummieren, und damit die Existenzmöglichkeiten von Muschelpopulationen noch weitaus stärker beeinträchtigt werden.

Danksagung. Die Autoren danken der Deutschen Forschungsgemeinschaft, die durch eine Sachbeihilfe im Rahmen des "Sonderprogramms Meeresverschmutzung" (Az.: Th 158/11) die Durchführung der Arbeit ermöglichte. 


\section{ZITIERTE LITERATUR}

Alderdice, D. F,, 1973. Factor combinations. In: Marine ecology. Ed. by O. Kinne. WileyInterscience, London. 1(3), 1659-1722.

- \& Thomson, J. A., 1974. Characteristic of some modified second-order orthogonal composite factorial designs. Tech. Rep. Fish. Res. Bd Can., 493, 1-70.

Bayne, B. L., 1965. Growth and delay of metamorphosis of the larvae of Mytilus edulis (L.). Ophelia 2, $1-47$.

Blackman, F. F. 1905. Optima and limiting factors. Ann. Bot. 19, 281-295.

Box, G. E. P. \& Wilson, K., 1951. On the experimental attainment of optimum conditions. C. r. statist. Soc. (B) 12, 1-38.

Brenko, M. H. \& Calabrese, A., 1969. The combined effects of salinity and temperature on the larvae of the mussel Mytilus edulis. Mar. Biol. 4, 224-226.

Brown, B. \& Ahsanullah, A., 1971. Effect of heavy metals on mortality and growth. Mar. Pollut. Bull. 2, 182-187.

Douglas-Wilson, I. 1972. Cadmium pollution and Itai-Itai disease. Lancet 1, 382-383.

Field, I. A., 1922. Biology and economic value of the mussel Mytilus edulis Bull. Bur. Fish., Wash. 38, $123-259$.

Förstner, U. \& Reineck, H.-E., 1974. Die Anreicherung von Spurenelementen in den rezenten Sedimenten eines Profilkerns aus der Deutschen Bucht. Senckenberg. marit. 6, 175-184.

Friberg, L., Piscator, M., Nordberg, G. F. \& Kjellström, T., 1974. Cadmium in the environment. CRC Press, Ohio, 284 pp.

Kobayashi, J., 1971. Relation between the "Itai-Itai" disease and the pollution of river water by cadmium from a mine. Adv. Wat. Pollut. Res. 1, 1-32.

Kremling, K., 1973. Voltametrische Messungen über die Verteilung von Zink, Cadmium, Blei und Kupfer in der Ostsee. Kieler Meeresforsch. 29, 77-84.

Lehnberg, W., 1974. Untersuchungen über die komplexe Wirkung ökologischer Faktoren auf den Gaswechsel von Delesseria sanguinea (Rhodophyta) aus der westlichen Ostsee unter Anwendung der "response surface"

Lewis, J. R., 1964. The ecology of rocky shores. The Engl. Univ. Press., London, 323 pp.

Lindesey, J. K., Alderdice, D. F. \& Pienaar, L. V., 1970. The analysis of non-linear models - the nonlinear response surface. J. Fish. Res. Bd Can. 27, 765-791.

- \& Sandnes, A. M., 1970. Program for the analysis of nonlinear response surfaces (extended version). Tech. Rep. Fish. Res. Bd Can. 173, 1-131.

Loosanoff, V. L. \& Davis, H. C., 1963. Rearing of bivalve mollusks. Adv. mar. Biol. 1, $1-136$.

Pavicić, J. \& Järvenpää, T., 1974. Cadmium toxicity in adults and early larval stages of the mussel Mytilus galloprovincialis Lam. In: Comparative studies of food and environmental contamination. Int. Atomenergiebehörde, Wien, 179-188.

Rosenberg, R. \& Costlow, jr., J. D., 1976. Synergistic effects of cadmium and salinity combined with constant cycling temperatures on the larval development of two estuarine crab species. Mar. Biol. 38, 291-303.

schlieper, C. \& Theede, H., 1975. Adaption of marine animals to abiotic environmental factors. (Russ.) Marine Biology (Vladivostok) 6, 3-25.

Theede, H., 1965. Vergleichende experimentelle Untersuchungen über die zelluläre Gefrierresistenz mariner Muscheln. Kieler Meerestorsch. 21, 153-166.

- 1974. Die Tierwelt. I. Okologie. In: Meereskunde der Ostsee. Hrsg. von L. Maagard \& G. Rheinheimer. Springer, Berlin, 171-188.

- 1979. Probleme der Anreicherung und Schadwirkung von Cadmium bei Meerestieren. Naturw. Rdsch., Stuttg. (Im Druck).

- Andersson, I. \& Lehnberg, W., 1979a. Cadmium in Mytilus edulis from German coastal waters. Meeresforsch. (Im Druck.)

- Scholz, N. \& Fischer, H., 1979b. Synergistic effects of temperature, salinity and cadmium on

Laomedea loveni. Mar. Biol. (In press). 
Westernhagen, H. von, Dethlefsen, V. \& Rosenthal, H., 1975. Combined effects of cadmium and salinity on development and survival of garpike eggs. Helgoländer wiss. Meeresunters. 27, 268to 282.

- Rosenthal, H. \& Sperling, K.-R., 1974. Combined effects of cadmium and salinity on development and survival of herring eggs. Helgoländer wiss. Meeresunters. 26, 416-433. 